\title{
KRULL DIMENSION AND TORSION RADICALS
}

\author{
A.K. Boyle and R.J. Serven
}

Let $R$ be a ring with Krull dimension $\alpha$ and let $\tau_{\alpha}$ be defined on a module $M_{R}$ by $\tau_{\alpha}(M)=\{m \in M:|m R|<\alpha\}$. Equivalent conditions for $\tau_{\alpha}$ to be a torsion radical are given. The relationship between $\tau_{\alpha}$-criticals and $\alpha$-criticals is also explored.

\section{Introduction and definitions}

This paper grew out of numerous discussions on the extension of results in Krull dimension by means of torsion radicals. If $R$ is a ring with Krull dimension $\alpha$, denoted $|R|=\alpha$, the natural candidate for a torsion radical in the Krull dimension setting is $\tau_{\alpha}$, where $\tau_{\alpha}$ is defined on the module $M_{R}$ by $\tau_{\alpha}(M)=\{m \in M:|m R|<\alpha\}$. This is always a preradical, but when $\alpha$ is a limit ordinal, this is not known to be a radical. This difficulty has been noted in several places (see for example [1] and [3]). The purpose of this paper is to determine when $\tau_{\alpha}$ is a torsion radical and when it is, to see how the torsion theoretic extensions compare to their Krull dimension models.

The torsion theory generated by the cyclics of Krull dimension less than $\alpha$ is denoted $T_{\alpha}$. Specifically for a right module $M$, $T_{\alpha}(M)=\{m \in M$ : every nonzero factor of $m R$ contains a nonzero submodule of Krull dimension less than $\alpha$ \}.

Received 24 September 1982. 
When $\tau_{\alpha}$ is a torsion radical, clearly $\tau_{\alpha}$ and $T_{\alpha}$ coincide. If the Krull dimension of $R$ is $\alpha$, then $R$ satisfies the descending chain condition on $T_{\alpha}$-closed submodules. A submodule $N \leq M$ is $T_{\alpha}$-closed in $M$ if $M / N$ is $T_{\alpha}$-torsionfree. Using this chain condition on $R$, we derive equivalent conditions, in the second section, for $\tau_{\alpha}$ to be a radical, where $\alpha=|R|$. When $R$ satisfies a stronger chain condition, namely when $R$ has a critical composition series, we show that $\tau_{\alpha}$ is always a torsion radical.

Unfortunately, even when $\tau_{\alpha}$ is a radical, there are major Krull dimension concepts which do not match-up with their torsion theoretic counterparts. In particular, $T_{\alpha}$-critical and $\alpha$-critical need not coincide. A nonzero $T_{\alpha}$-torsionfree module is called $T_{\alpha}$-critical if for $0 \neq N \leq M, M / N$ is $T_{\alpha}$-torsion. This module is a-critical if, in addition, $M$ has Krull dimension and $|M / N|<\alpha$. When $\tau_{\alpha}$ is a radical, $T_{\alpha}$-critical and $\alpha$-critical coincide for cyclic modules but need not coincide for modules with larger generating sets. In the third section, we consider when $T_{\alpha}$-criticals which have Krull dimension are $\alpha$-critical. We show that this problem is interlaced with the existence of critical composition series and maximal submodules of Krull dimension less than $\alpha$.

All rings in this paper are associative with unit and all modules are unital. Module will mean right module. A certain familiarity with the definitions and basic results concerning Krull dimension, as given in [4], and torsion theory, as given in [7], is assumed. If $M$ is a module with Krull dimension $\beta$, we write $|M|=\beta$. Unless otherwise noted, the ring $R$ is always assumed to have Krull dimension $\alpha$.

\section{When $\tau_{\alpha}$ is a radical}

Let $M$ be a right $R$-module with Krull dimension $\alpha$. Then $M \neq 0$ is called $\alpha$-smooth if $M$ is $T_{\alpha}$-torsionfree; that is, if every nonzero submodule has Krull dimension $\alpha$. We say that a module $M$, with 
$|M|=\alpha$, has enough smooth factors if for any submodule $N$ of $M$ with $|M / N|=\alpha$, there exists a submodule $N^{\prime}$ of $M$ containing $N$ such that $M / N^{\prime}$ is $\alpha$-smooth.

LEMMA 2.1. Let $M$ be $T_{\alpha}$-critical with Krull dimension. Then $M$ is a-critical if and only if $M$ has enough smooth factors.

Proof. If $M$ is $\alpha$-critical and $N \leq M$ with $|M / N|=\alpha$, then $N=0$. Since $M$ is $\alpha$-smooth, $M$ has enough smooth factors.

Conversely, if $M$ has enough smooth factors, $N \leq M$ and $|M / N|=\alpha$, then there exists $N \leq N^{\prime} \leq M$ such that $M / N^{\prime}$ is $\alpha$-smooth. Since $M$ is $T_{\alpha}$-critical, it follows that $N^{\prime}=0$. Hence $M$ is $\alpha$-critical.

A collection $L$ of right ideals is said to be cofinally finite if every element of $L$ contains a finitely generated element of $L$.

We note that, by $[7,3.4], \tau_{\alpha}$ is a torsion radical if and only if $M_{\alpha}=\left\{I \leq R_{R}:|R / I|<\alpha\right\}$ is a topology.

THEOREM 2.2. Let $R$ be a ring with Krull dimension $\alpha$. The following statements are equivalent:

(1) $\tau_{\alpha}$ is a radical;

(2) every cyclic module with Krull dimension a has enough smooth factors;

(3) every cyclic module with Krull dimension a has an a-critical factor module;

(4) $M_{\alpha}$ is cofinally finite.

Proof. (1) $\Rightarrow(2)$. It suffices to show that every cyclic module with Krull dimension $\alpha$ has a smooth factor module. If $M=m R$ has Krull dimension $\alpha$, then $m \neq \tau_{\alpha}(M)$. Thus $M / \tau_{\alpha}(M) \neq 0$. By (1), $M / \tau_{\alpha}(M)$ is $\alpha-s$ mooth.

(2) $\Rightarrow$ (3). Let $|R / I|=\alpha$ for $I$ a right ideal of $R$. Then by (2), there exists $I \leq N \leq R_{R}$ such that $R / N$ is $\alpha$-smooth. By [6, 1.4], $R$ has the ascending chain condition on $T_{\alpha}$-closed submodules. Thus there 
exists a maximal proper $T_{\alpha}$-closed right ideal $N^{\prime}$ containing $N$. Then $R / N^{\prime}$ is $T_{\alpha}$-critical and, hence, $\alpha$-critical by Lemma 2.1 .

(3) $\Rightarrow(1)$. Since $\tau_{\alpha}(M) \leq T_{\alpha}(M)$ for any right $R$-module $M$, it suffices to show that if $m \in T_{\alpha}(M)$, then $|m R|<\alpha$. If not, then $m R$ has an $\alpha$-critical factor module, by (3), which is necessarily a nonzero $\alpha$-smooth factor module. This contradicts the fact that $m R$ is $T_{\alpha}$-torsion.

$(1) \Rightarrow(4)$. By $[6,1.4], R$ has the ascending chain condition on $T_{\alpha}$-closed submodules. The result now follows from $[8,1.2$ and 1.5$]$.

(4) $\Rightarrow(1)$. Since $\tau_{\alpha}$ is always a preradical, it suffices to show that $\tau_{\alpha}\left(M / \tau_{\alpha}(M)\right)=0$ for all right $R$-modules $M$. Let $\bar{m} \in \tau_{\alpha}\left(M / \tau_{\alpha}(M)\right)$. Then there exists $I \in M_{\alpha}$ such that $m I \leq \tau_{\alpha}(M)$. By (4) we may assume that $I$ is finitely generated. Then $m I$ is finitely generated and $|m I|<\alpha$. Furthermore $I \in M_{\alpha}$ implies that $|R / I|<\alpha$ and hence that $|m R / m I|<\alpha$. By [4,1.1], $|m R|<\alpha$. Thus $m \in \tau_{\alpha}(M)$ and $\bar{m}=0$.

The following corollaries provide specific situations in which $\tau_{\alpha}$ is a radical.

COROLLARY 2.3. Let $R$ be a ring with Krull dimension $\alpha$.

(1) If $R$ is right noetherion, then $\tau_{\alpha}$ is a radical.

(2) If $\alpha$ is a nonlimit ordinal, then $\tau_{\alpha}$ is a radical.

Proof. (1) This is a direct consequence of Theorem 2.2 (4).

(2) Let $M$ be a cyclic module with Krull dimension $\alpha$. By [4, 4.2], $M$ has a maximal submodule $N$ of Krull dimension less than or equal to $\alpha-1$. Clearly $M / N$ is $\alpha$-smooth. The result now follows from Theorem 2.2 (2).

Let $R$ be a ring with Krull dimension $\alpha$ and let $N$ denote the prime radical of $R$. Then $N$ is said to be right weakly ideal invariant if $|N / I N|<\alpha$ for any $I \in M_{\alpha}$. Beachy [1] observed that for rings that 
have this property, $\tau_{\alpha}$ is a radical.

COROLLARY 2.4. Let $R$ be a ring with Krulz dimension a. If the prime radical of $R$ is weakly ideal invariant, then $\tau_{\alpha}$ is a radical.

Proof. By [5, Corollary 5], if $I \in M_{\alpha}$ then there exists $c R \leq I$ such that $c R \in M_{\alpha}$. Thus $M_{\alpha}$ is cofinally finite. Apply Theorem 2.2 (4).

Let $M$ be a module with Krull dimension. Then $M$ has a critical composition series if there exists a finite chain

$$
M=K_{n}>K_{n-1}>\ldots>K_{0}=0
$$

with $K_{i} / K_{i-1} \alpha_{i}$-critical for all $i, I \leq i \leq n$ and $\alpha_{n} \geq \alpha_{n-1} \geq \ldots \geq \alpha_{1}$.

PROPOSITION 2.5. Let $M$ be a module with a critical composition series. If $N \leq M$ such that $M / N$ is $T_{B}$-torsion, where $B=|M|$, then $|M / N|<\beta$.

Proof. Let $M=K_{n}>K_{n-1}>\ldots>K_{0}=0$ denote a critical composition series for $M$ and let $j$ denote the largest integer such that $\left|K_{j}\right|<\beta$. Then $|M / N|=\sup \left\{\left|M /\left(N+K_{j}\right)\right|,\left|\left(N+K_{j}\right) / N\right|\right\}$. Since $\left|\left(N+K_{j}\right) / N\right|=\left|K_{j} /\left(N \cap K_{j}\right)\right|<\beta$, it suffices to show that $\left|M /\left(N+K{ }_{j}\right)\right|<\beta$. Hence we can consider the case when $N \supset K_{j}$ and work in the smooth module $M / K_{j}$. Without loss of generality, we will assume that $K_{j}=0$ and, hence, that $M$ is $\beta$-smooth.

The proof is by induction on the length of the critical composition series for $M$. If $M$ is $\beta$-critical and $M / N$ is $T_{B}$-torsion, then $N \neq 0$. Thus $|M / N|<\beta$. Assume that $n>1$ and that the result holds for modules which have a $B$-critical composition series of length $n-1$ or less. If $N \geq K_{1}$, then $N / K_{1} \leq M / K_{1}$ and $\left(M / K_{1}\right) /\left(N / K_{1}\right) \cong M / N$ is $T_{B}$-torsion. Then $|M / N|<\beta$ since $M / K_{1}$ has a $B$-critical composition series of length $n-1$. Thus we may assume that $N \cap K_{1}<K_{1}$. If 
$N \cap K_{1} \neq 0$, then $\left|K_{1} /\left(N \cap K_{1}\right)\right|<\beta$, since $K_{1}$ is $\beta$-critical. Thus $\left|\left(N+K_{1}\right) / N\right|<\beta$. Since $N \leq N+K_{1}, M /\left(N+K_{1}\right)$ is $T_{\beta}$-torsion and hence $\left|M /\left(N+K_{1}\right)\right|<\beta$ by induction. Therefore, $|M / N|<\beta$. Thus we may assume that $N \cap K_{1}=0$. However, then there exists an injection of $K_{1} \cong\left(N+K_{1}\right) / N$ into $M / N$. This is a contradiction since $M / N$ is $T_{B}$-torsion and $0 \neq K_{1}$ is $B$-smooth. Hence $|M / N|<B$.

COROLLARY 2.6. Let $R$ be a ring with a critical composition series and $|R|=\alpha$. Then $\tau_{\alpha}$ is a radical.

Proof. It suffices to show that if $T_{\alpha}(R / I)=R / I$, where $I \leq R_{R}$, then $|R / I|<\alpha$. The result now follows from Proposition 2.5.

\section{3. $\tau_{\alpha}$-criticals and $\alpha$-criticals}

When $R$ is $\alpha$-smooth, the converses of Proposition 2.5 and Corollary 2.6 are tempting since by $[2,1.2]$ and $[6,1.4]$ every $\alpha$-smooth module has a descending chain in which the factors are $\tau_{\alpha}$-critical, that is, a $\tau_{\alpha}$-composition series. Regrettably, this need not be a critical composition series since a $\tau_{\alpha}$-critical is not necessarily $\alpha$-critical. See, for example, [3]. The major impediment is the inability to construct maximal submodules of Krull dimension less than $\alpha$. In this section we discuss the relationship between these concepts.

PROPOSITION 3.1. A module $M$ with Krull dimension $\alpha$ has a maximal submodule of Krull dimension less than $\alpha$ if and only if every submodule with Krull dimension $\alpha$ has an a-smooth factor.

Proof. Let $T$ denote the maximal submodule of Krull dimension less than $\alpha$. Then $M / T$ is $\alpha$-smooth. If $N \leq M$ has $|N|=\alpha$, then $N /(N \cap T) \cong(N+T) / T \leq M / T$ is $\alpha$-smooth. Note that $|N|=\alpha$ implies $N /(N \cap T) \neq 0$.

Conversely, let $T$ denote the intersection of all $T_{\alpha}$-closed submodules of $M$. Then $M / T$ is $\alpha$-smooth. If $|T|=\alpha$, then by hypothesis, $T$ has a submodule $W$ with $T / W \alpha$-smooth. This implies that 
$M / W$ is $\alpha$-smooth and hence $W$ is $T_{\alpha}$-closed. Then $T \leq W$, so $T / W=0$, a contradiction. Thus $|T|<\alpha$ and $T$ is the maximal submodule of Krull dimension less than $\alpha$.

The existence of maximal submodules of Krull dimension less than $\alpha$ in factors of $M$ enables us to construct a critical composition series for $M / \tau_{\alpha}(M)$

THEOREM 3.2. Let $R$ be a ring with $|R|=\alpha$ and let $M$ be a right R-module with $|M|=\alpha$. The following statements are equivalent:

(1) $M / \tau_{\alpha}(M)$ has a critical composition series and $\left|\tau_{\alpha}(M)\right|<\alpha$;

(2) every factor module of $M$ has a maximal submodule of Krull dimension less than $\alpha$.

Proof. (1) $\Rightarrow(2)$. Let $N \leq M$. If $|M / N|<\alpha$, we are done. Suppose $|M / N|=\alpha$ and let $S / N=\tau_{\alpha}(M / N)$. If $S=M$, then $S \geq \tau_{\alpha}(M)$. If $S \neq M$, then $M / S$ is $\alpha$-smooth which implies that $S \geq \tau_{\alpha}(M)$. If $S=\tau_{\alpha}(M)$, then $|S|<\alpha$ and $S / N$ is the desired submodule. Thus we may assume $S>\tau_{\alpha}(M)$. Since $M / \tau_{\alpha}(M)$ has a critical composition series, every submodule has one and so, in particular, $S / \tau_{\alpha}(M)$ has a critical composition series. Now $S /\left(N+\tau_{\alpha}(M)\right)$ is $T_{\alpha}$-torsion and, hence, Proposition 2.5 applied to $S / \tau_{\alpha}(M)$ gives $\left|S /\left(N+\tau_{\alpha}(M)\right)\right|<\alpha$. Since $\left|\left(N+\tau_{\alpha}(M)\right) / N\right|=\left|\tau_{\alpha}(M) /\left(\tau_{\alpha}(M) \cap N\right)\right| \leq\left|\tau_{\alpha}(M)\right|<\alpha$, then $|S / N|<\alpha$. Thus $S / N$ is the desired submodule of $M / N$.

(2) $\Rightarrow(1)$. By hypothesis $M$ has a maximal submodule $N$ of Krull dimension less than $\alpha$. Necessarily, $N=\tau_{\alpha}(M)$. Then $M / \tau_{\alpha}(M)$ is $\alpha$-smooth. By $[6,1.4]$ and $[2,1.2], M / \tau_{\alpha}(M)$ has a $T_{\alpha}$-composition series. Let $M=K_{n}>K_{n-1}>\ldots>K_{0}=\tau_{\alpha}(M)$ denote such a series. If $N / K_{i-1}<K_{i} / K_{i-1}$ such that $\left|K_{i} / N\right|=\alpha$, then $M / N$ has a maximal submodule $S / N$ of Krull dimension less than $\alpha$. By Proposition 3.1, $K_{i} / N$ has an $\alpha$-smooth factor. Hence $K_{i} / K_{i-1}$ has enough smooth factors. Thus $k_{i} / k_{i-1}$ is a-critical by Lemma 2.1 . 
In particular, for a ring with Krull dimension $\alpha, R / \mathrm{\tau}_{\alpha}(R)$ has a critical composition series and $\left|\tau_{\alpha}(R)\right|<\alpha$ if and only if every cyclic module has a maximal submodule of Krull dimension less than $\alpha$. In this case, every cyclic module with Krull dimension $\alpha$ has enough smooth factors. By Theorem 2.2 (2), we have the following.

COROLLARY 3.3. Let $R$ be a ring with Krull dimension $\alpha$. If $\left|\tau_{\alpha}(R)\right|<\alpha$ and $R / \tau_{\alpha}(R)$ has a critical composition series, then $\tau_{\alpha}$ is a radical.

If $\alpha$ is not a limit ordinal, then by [4, 4.1] every module with Krull dimension $\alpha$ has a maximal submodule of Krull dimension less than $\alpha$. Thus, by Theorem 3.2, if $|M|=\alpha$, then $M / \tau_{\alpha}(M)$ has a critical composition series. Furthermore, as the next result shows, every $T_{\alpha}$-critical which has Krull dimension is $\alpha$-critical.

COROLLARY 3.4. Let $M$ be a right R-module with $|M|=\alpha$. If $M / \tau_{\alpha}(M)$ has a critical composition series and $\left|\tau_{\alpha}(M)\right|<\alpha$, then every $T_{\alpha}$-critical which is contained in a factor of $M$ is a-critical.

Proof. If $C / N$ is a $T_{\alpha}$-critical submodule of $M / N$, then $C / N$ can be embedded in $(M / N) / T_{\alpha}(M / N)$. Thus we may assume that $M / N$ is $\alpha-s$ mooth.

Every factor module of $M$ has a maximal submodule of Krull dimension less than $\alpha$ by Theorem 3.2. Hence the same is true of $M / N$. By Theorem 3.2, $M / N$ has a critical composition series and this implies that $C / N$ has a critical composition series. The result now follows from Proposition 2.5 .

COROLLARY 3.5. Let $R$ be a ring with Krull dimension $\alpha$ and let $M$ be an $\alpha$-smooth module with Krull dimension. The following are equivalent:

(1) $M$ has a critical composition series;

(2) every factor of $M$ has a maximal submodule of Krull dimension less than $\alpha$;

(3) every $T_{\alpha}$-critical submodule of a factor of $M$ is 
$\alpha$-critical.

COROLLARY 3.6. Let $R$ be a ring with Krull dimension $\alpha$.

(1) If every module with Krull dimension a has a maximal submodule of Krull dimension less than $\alpha$, then every $T_{\alpha}$-critical module which has Krull dimension is a-critical.

(2) Every $T_{\alpha}$-critical module with Krull dimension is $\alpha$-critical if and only if every $\alpha$-smooth module with Krull dimension has a critical composition series.

REMARK 1 . It would seem appropriate to include an example of a ring with Krull dimension $\alpha$ for which $\tau_{\alpha}$ is not a torsion radical.

Unfortunately, the authors know of no such example. The above results grew out of attempts to construct such an example and demonstrate the difficulties which one encounters.

REMARK 2. Although there are examples of $T_{\alpha}$-critical $R$-modules with Krull dimension which are not $\alpha$-critical, the ring $R$ does not have Krull dimension. It would be interesting to know an example where $R$ has Krull dimension.

\section{References}

[1] John A. Beachy, "Weak ideal invariance and orders in Artinian rings: Corrigendum", Bull. Austral. Math. Soc. 25 (1982), 317-320.

[2] Oscar Goldman, "Elements of noncommutative arithmetic I", J. Algebra 35 (1975), 308-341.

[3] Robert Gordon, "Gabriel and Krull dimension", Ring theory, 241-295 (Proc. Ring Theory Conf., Oklahoma, 1973. Lecture Notes in Pure and Applied Mathematics, 7. Marcel Dekker, New York, 1974).

[4] Robert Gordon and J.C. Robson, Krull dimension (American Mathematical Society Memoirs, 133. American Mathematical Society, Providence, Rhode Island, 1973).

[5] Gunter Krause, T.H. Lenagan and J.T. Stafford, "Ideal invariance and Artinian quotient rings", J. Algebra 55 (1978), 145-154. 
[6] Robert W. Miller and Mark L. Teply, "The descending chain condition relative to a torsion theory", Pacific J. Math. 83 (1979), 207-219.

[7] Bo Stenström, Rings and modules of quotients (Lecture Notes in Mathematics, 237. Springer-Verlag, Berlin, Heidelberg, New York, 1971 ).

[8] Mark L. Teply, "Torsionfree injective modules", Pacific J. Math. 28 (1969), 441-453.

Department of Mathematics,

University of Wisconsin-Mi I waukee,

Mi I waukee,

Wisconsin 53201,

USA. 